\title{
Monitoring Drug Release from Electrospun Fibers Using an In Situ Fiber-Optic System
}

\author{
Salem Seif ${ }^{1,2}$, Florian Graef ${ }^{3}$, Sarah Gordon ${ }^{3}$, and Maike Windbergs ${ }^{1,2,3, *}$ \\ ${ }^{1}$ Saarland University, Department of Biopharmaceutics and Pharmaceutical Technology, \\ Saarbruecken, Germany \\ ${ }^{2}$ PharmBioTec GmbH, Saarbruecken, Germany \\ ${ }^{3} \mathrm{Helmholtz}$ Centre for Infection Research (HZI) and Helmholtz Institute for Pharmaceutical Research \\ Saarland (HIPS), Department of Drug Delivery (DDEL), Saarbruecken, Germany
}

e-mail: m.windbergs@mx.uni-saarland.de

\section{ABSTRACT}

Electrospun fiber mats are currently gaining attention as advanced drug delivery systems. Dissolution testing for such systems is generally performed in small vials by immersing the fiber mats in buffered solutions. Defined aliquots of dissolution medium are withdrawn at predefined time points, and the dissolved drug is quantified. However, this procedure is associated with several drawbacks. The method is not automated, and as such requires manual sampling, which potentially leads to inaccuracies particularly in frequent sampling intervals as required for characterization of rapid drug release. Further, the sheet-like fiber mats tend to partially fold upon contact with the dissolution medium, which may potentially affect the release kinetics and reproducibility of the acquired release data.

In this study, we investigated the application of a fully automated fiber-optics based dissolution testing system for in situ monitoring of drug release from electrospun fiber mats. Electrospun poly(vinyl alcohol) fibers loaded with lysozyme were used as a model system. To prevent folding of the fiber mats and ensure a fixed position in the dissolution vessel throughout the experiment, a flexible adapter was developed to allow for the attachment of the fiber mats to the vessel walls. Lysozyme release from the fiber mats was compared with the release from cast films with the same composition. Even though the release processes were rather fast and differences in release kinetics of the two systems were marginal, the fiber-optics based dissolution setup allowed for the successful detection of released protein in both cases. The present study, therefore, highlights the potential for the utilization of fully automated fiber-optics based dissolution testing systems for advanced in situ monitoring of drug release from electrospun fibers.

KEYWORDS: Fiber optic; electrospun fiber mats; dissolution.

\section{INTRODUCTION}

lectrospinning is a highly versatile technique that enables the production of ultrafine fibers based on polymeric solutions. In recent years, the interest in electrospinning has significantly increased as the technique provides promising applications in many scientific fields (1). A special interest has been directed towards applications of electrospun systems for drug delivery (2). Electrospun fibers provide unique characteristics such as high surface-to-volume ratio, and depending on the polymer, tailor-made release kinetics for embedded drugs $(2,3)$. Additionally, electrospinning provides the ability to incorporate different types of active pharmaceutical ingredients varying in origin, physicochemical properties, and molecular size (4). Conventional dissolution experiments for drug-loaded electrospun fibers are conducted in small vials by immersing a preweighed sample in dissolution medium at physiological $\mathrm{pH}$ and temperature. At predetermined time points, defined aliquots are then withdrawn for drug quantification and replaced with fresh dissolution medium (5). However, this dissolution testing procedure is associated with several technical drawbacks. First, as the method is not automated, manual sampling is required. This creates limitations in terms of the possible overall duration of such experiments, and the accuracy of data obtained from frequent sampling as required for the analysis of rapid drug release processes. Furthermore, as the fiber mats are flexible sheet-like structures, irregular partial folding or creasing of the mats tends to occur when introduced to the aqueous medium. This change of conformation may, in turn, affect release kinetics and, potentially, the reproducibility of acquired data.

As an alternative to conventional dissolution testing involving the withdrawal of medium aliquots for drug

${ }^{*}$ Corresponding author. 
quantification, systems based on optical fibers were introduced allowing for continuous in situ sampling directly in the dissolution medium (6). Such systems allow for the direct UV measurement of dissolved drug within samples, and therefore a real-time dissolution profile can be obtained (6). The initial report regarding the application of such systems for the analysis of drug release from tablets was published by Josefson et al. in 1988 (7). Since then, technical progress has paved the way for standardized optical fiber probes with the opportunity for the concentration assessment of dissolved drug within several individual vessels $(8,9)$. The automation of this process facilitates reproducibility and convenience, while the ability to measure concentration accurately at high frequency allows for improved detection and a better understanding of release kinetics within short time frames. However, even though the suitability of such a method has already been proven, the assessment of release from thin flexible systems like fiber mats provides additional challenges. Due to their low weight and large surface area, the mats may remain suspended within or on the surface of the dissolution medium throughout the course of the experiment rather than sinking to the bottom of the dissolution vessel, thus distorting reliable data acquisition. Furthermore, the aforementioned tendency of such sheet-like structures to assume a partially folded conformation upon contact with liquids is prone to affect the release kinetics of mat-embedded drugs.

In this study, we investigated the suitability of a fully automated dissolution testing system based on fiber optics for in situ monitoring of drug release from electrospun fiber mats in comparison with cast films. To prevent folding of the fiber mats and films in the dissolution medium, we developed an adapter allowing for the attachment of the fiber mats and films to the wall of the dissolution vessels.

\section{MATERIALS AND METHODS}

\section{Materials}

Poly(vinyl alcohol) (PVA; Mw 85,000-124,000, >99\% hydrolyzed) and lysozyme from chicken egg white ( 70,000 U/mg) were purchased from Sigma-Aldrich (Steinheim, Germany). Acetic acid was obtained from Fluka (Buchs, Switzerland).

\section{Methods}

\section{Fabrication of Electrospun Fiber Mats}

PVA was dissolved in purified water $(10 \% \mathrm{w} / \mathrm{v})$ with acetic acid $(1 \% \mathrm{v} / \mathrm{v})$. After adding lysozyme $(6 \% \mathrm{w} / \mathrm{w}$ drug/ polymer), the solution was spun using an electrospinning setup consisting of a syringe pump system (New Era
Pump Systems, Inc., USA) and a high voltage power supply (Acopian, USA). The polymeric solution was pumped through a syringe nozzle with a flow rate of $0.5 \mathrm{~mL} / \mathrm{h}$ while a high voltage of $20 \mathrm{kV}$ was applied. The formed electrospun fibers were deposited on a metal collector located at a distance of $14 \mathrm{~cm}$ from the nozzle. After electrospinning, the lysozyme-PVA fibers were stored in a desiccator for $24 \mathrm{~h}$ before further analysis.

\section{Fabrication of Cast Films}

Film-casting was performed by pipetting a defined amount of the lysozyme-PVA solution into a Petri dish, followed by homogenous distribution of the solution on the dish surface. The Petri dishes containing the lysozyme-PVA solutions were left at room temperature for at least $24 \mathrm{~h}$ to ensure complete solvent evaporation before being stored in the desiccator.

\section{Fourier-Transform Infrared Spectroscopy}

An attenuated total reflectance (ATR) unit was used to record Fourier-transform infrared (IR) spectroscopy spectra (Spectrometer 400 ATR-IR, Perkin Elmer, USA). Spectra of raw substances, cast films, and electrospun fibers were collected in the range of $550-4000 \mathrm{~cm}^{-1}$. All spectra were recorded at least in triplicate.

\section{Scanning Electron Microscopy}

Samples of the electrospun fibers or cast films were mounted on aluminum stubs using double-sided carbon discs and sputtered with a thin layer of gold prior to analysis (Sputter Coater Q150R ES, Quorum Technologies Ltd., UK). The scanning electron microscopy (SEM) analysis was performed at an accelerating voltage of $5 \mathrm{kV}$ using a Zeiss EVO HD 15 SEM (Carl Zeiss AG, Oberkochen, Germany).

\section{In Vitro Drug Release}

In vitro drug release experiments were performed using an in situ fiber-optic monitoring system, the $\mu \mathrm{DISS}$ Profiler, supported by Au PRO software (version 5.1.0.0, Pion Inc., Woburn, MA, USA). The fiber-optic probes were operated with tips of $20-\mathrm{mm}$ path length. Prior to the experiments, each channel was calibrated with its own standard curve prepared from multiple additions of a fresh solution of lysozyme in phosphate buffered saline (PBS) buffer. Punches of electrospun fiber mats and cast films were prepared (16-mm diameter), and drug release experiments were carried out in $15 \mathrm{~mL}$ PBS pH 7.4 at $37{ }^{\circ} \mathrm{C}$ with magnetic stirring at $100 \mathrm{rpm}$. UV absorption $(280 \mathrm{~nm})$ was measured at a time interval of $10 \mathrm{sec}$ for the first $5 \mathrm{~min}$, followed by measurements at $30-\mathrm{sec}$ intervals for the following $10 \mathrm{~min}$. After this initial period, measurements were performed every $60 \mathrm{sec}$. Release experiments were performed at least in triplicate.

Dissolution 


\section{RESULTS AND DISCUSSION}

\section{Fabrication and Characterization of}

\section{Electrospun Fibers and Cast Films}

To investigate the applicability of an in situ fiberoptic monitoring system for testing drug release from electrospun fibers, lysozyme was used as a model drug and encapsulated within electrospun fibers based on PVA. For comparison, cast films based on the same solution used for electrospinning were prepared. Despite the identical composition of both polymeric systems, a clear difference in the visual appearance could be noticed. The electrospun fiber mats exhibited an opaque white color (Figure $1 \mathrm{~A}$ ) that can be attributed to the ultrafine substructure of the fiber network (Figure 1B) (10). In contrast, the cast films appeared transparent with a smooth surface (Figure $1 C, D$ ). Both samples were prepared as circular punches with the same diameter and therefore the same apparent surface area. However, the ultrafine substructure of the electrospun fibers (individual fiber diameters approximately $500 \mathrm{~nm}$ ) increases the actual surface area of these samples, which can be expected to have an impact on the release kinetics of embedded drugs.

To elucidate any potential chemical interactions as a result of sample fabrication, ATR-IR spectra of pure compounds, fiber mats, and cast films were acquired. When comparing the ATR-IR spectra of the pure compounds to the spectra of the drug-loaded systems, neither the appearance of novel peaks nor the extinction of initial peaks (indicative of a chemical interaction as a result of sample preparation or instability) occurred (Figure 2). Further, comparison of

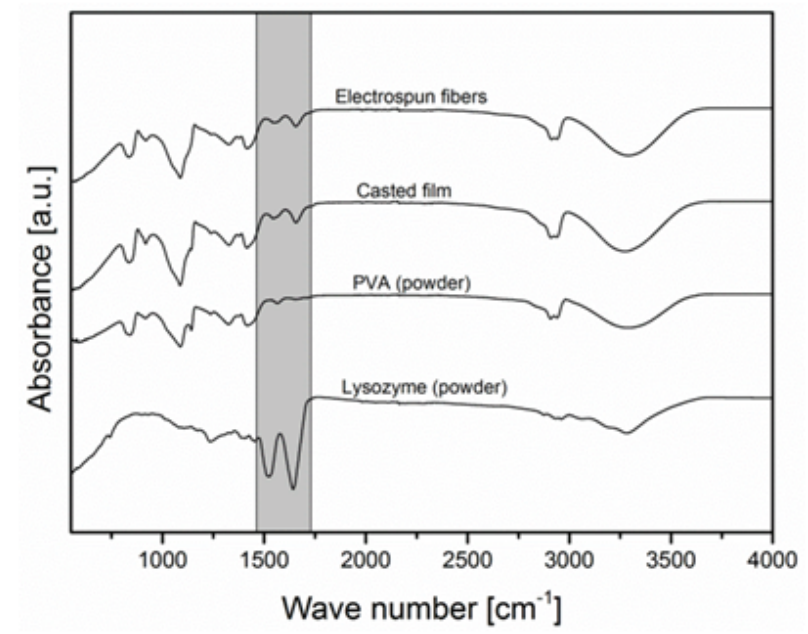

Figure 2. ATR-IR spectra of pure compounds, electrospun fiber mats, and cast films.

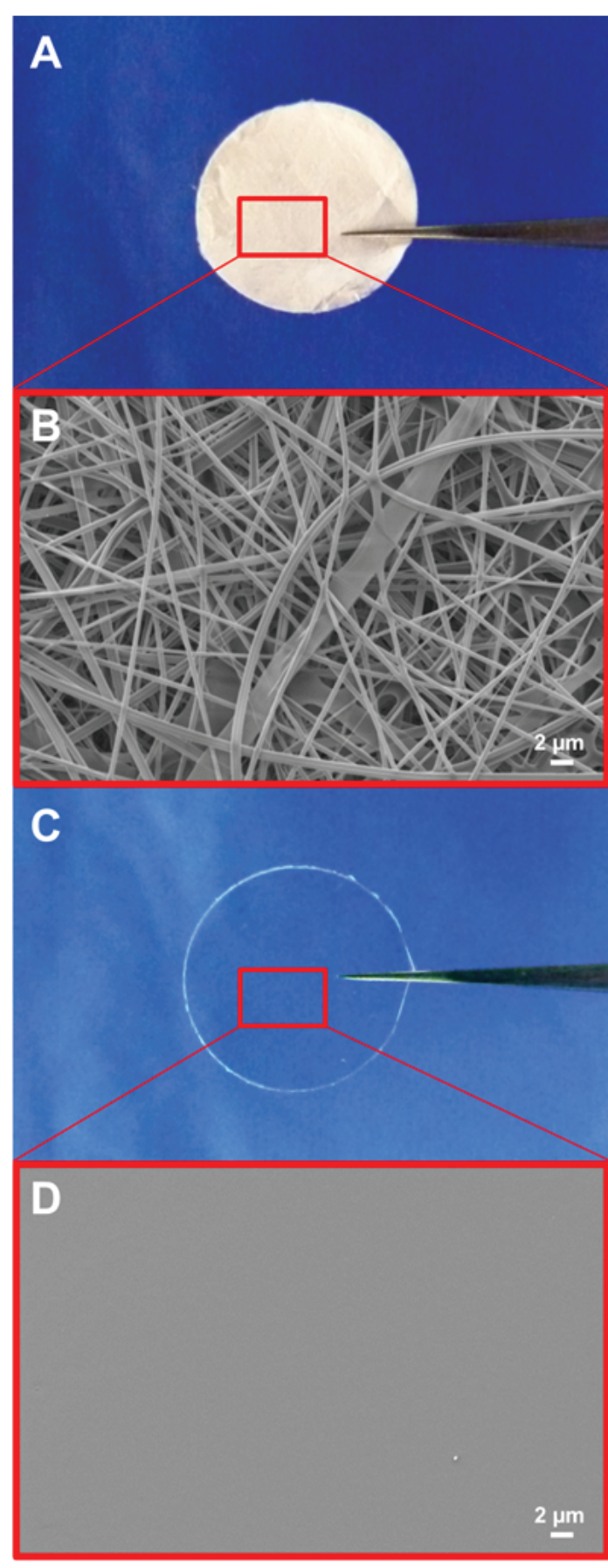

Figure 1. (A) Surface morphology of the electrospun fibers, (B) SEM image of the electrospun fibers, (C) surface morphology of a cast film, (D) SEM image of a cast film.

the spectra of films and fiber mats does not reveal any differences due to the respective fabrication technique. In addition, the incorporation of lysozyme into the fibers as well as into the films can be detected by the amide I peak $\left(1650 \mathrm{~cm}^{-1}\right)$. 


\section{Conventional Release Experiments with \\ Electrospun Fibers}

Conventional drug release experiments are performed by immersing the drug-loaded dosage form in buffer and quantifying the concentration of the released drug at predetermined time points. Although thin polymeric dosage forms such as electrospun fiber mats, films, or transdermal patches can potentially be investigated using this method, the thin and flexible structure of these dosage forms make them susceptible to folding upon immersion in the release medium as illustrated in Figure 3. This folding process is likely to decrease the surface area of the investigated sample, leading to inaccurate assessments of the release kinetics.

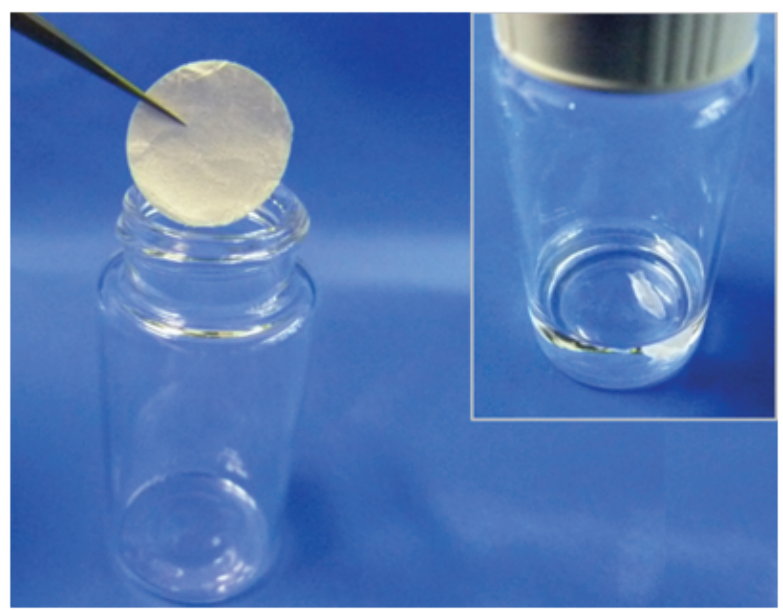

Figure 3. Electrospun fiber mat with a glass vial for conventional drug release testing before immersion in dissolution medium and in dissolution medium (magnification).

This phenomenon necessitates the preservation of the flat structure of the sample during the release experiment for improved accuracy and reproducibility. Indeed, dissolution testing for transdermal patches as described in the pharmacopeia (11) requires the fixation of the sample by using a strip of double-sided adhesive tape or an extraction cell. The same concept has been presented for polymeric films $(12,13)$. For electrospun fibers, however, drug release experiments are generally conducted by simply immersing the fiber mats in buffer without any fixation of the sample (5), even though there are few reports where the issue of sample folding was addressed. For instance, a modified Finn chamber was used for investigating drug release from electrospun fibers, hence keeping the sample from folding during the release experiments (14). In the present study, a novel flexible adapter was designed. The aim was to keep the sample fixed in a certain position during the release study, thus preventing migration and folding.
The designed adapter was composed of commercially available silicone and a nylon-based net (Figure 4). The mechanical flexibility of silicone allowed for convenient use, while the net provided a suitable support and, at the same time, unrestricted diffusion of the release medium and released drug. Prior to the release experiments, interactions of net material and drug were experimentally excluded (data not shown).

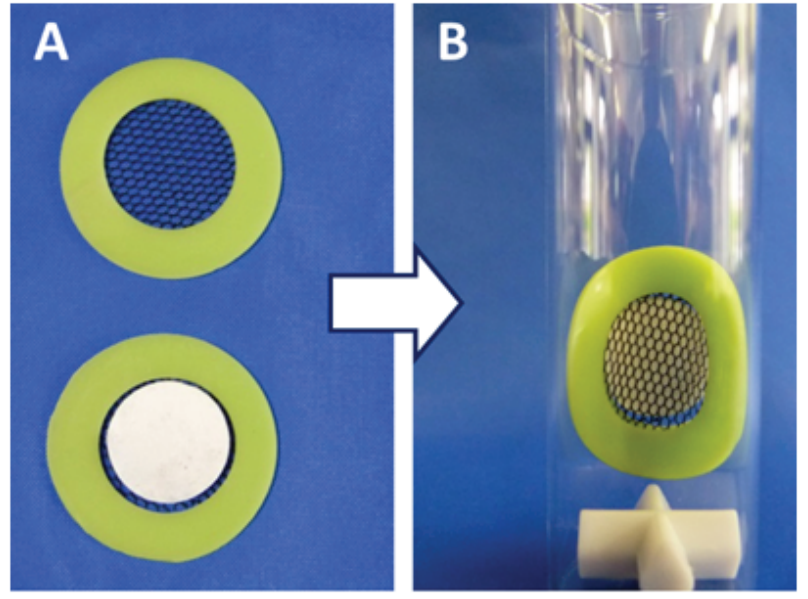

Figure 4. Sample mounting using the designed flexible adapter (A) Adapter without (top) and with (bottom) electrospun mat; (B) adapter with fiber mat installed within the dissolution vessel.

\section{In Vitro Drug Release with the Fiber-Optic System}

Release experiments in PBS pH 7.4 were performed with the electrospun fiber mats as well as with the cast films. The samples were mounted using the designed adapter, which assured holding the sample flat in position and prevented undesired sample folding (Figure 4). During the experiment, the dissolution vials were tightly sealed to prevent volume change due to medium evaporation. Each fiber-optic channel (corresponding to a separate dissolution vessel) was calibrated with its own standard curve prior to experimentation, and a very good linearity was achieved as shown in the representative calibration curve (Figure 5A). The theoretical drug content in the fiber mats and films was calculated based on the solid mass of lysozyme compared with the solid mass of the polymer. Thus, solvent evaporation upon electrospinning (or film casting) did not affect the lysozyme/polymer mass ratio. These calculations were successfully proofed by drug content analysis after destroying fiber mats and films. Complete drug release from the electrospun fibers as well as from the cast films was observed after approximately 20 min, which can be expected due to the hydrophilic nature of the drug and the polymeric fiber material. The results of the release experiments are shown in Figure $5 \mathrm{~B}$, and the data are represented as mean $\pm \operatorname{SD}(n=3)$. 

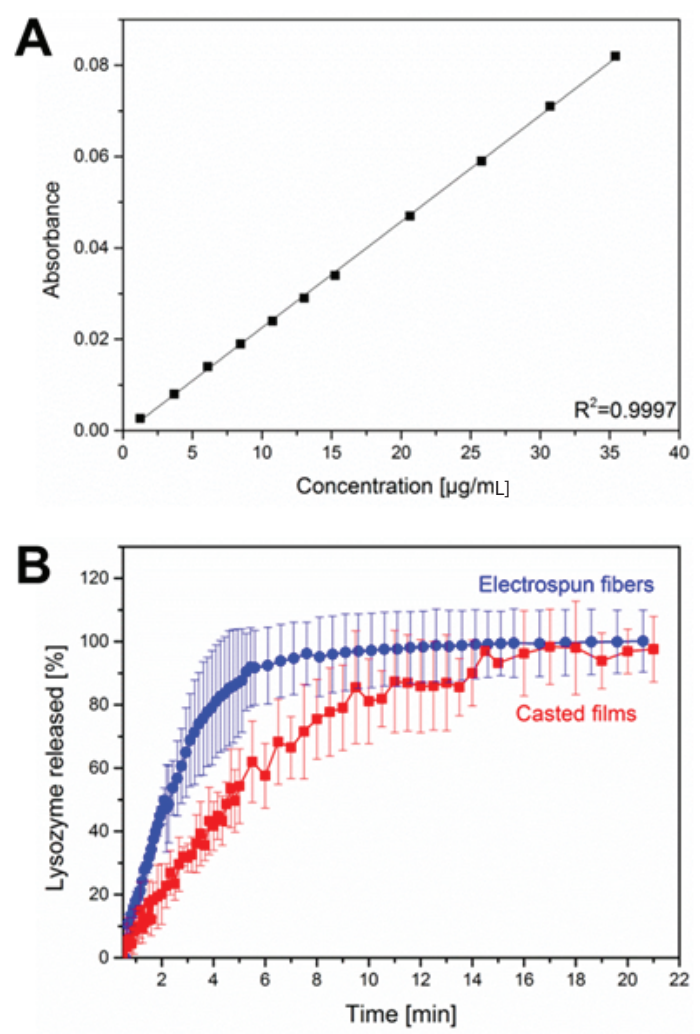

Figure 5. (A) Representative calibration curve and (B) lysozyme release from fiber mats as well as from films (mean $\pm S D, n=3$ ).

The frequent sampling intervals provided a deeper insight into the release process from the electrospun fibers as well as from the cast films. In this context, we can see that lysozyme was released faster from the electrospun fibers than from the cast films (Figure 5B). This can be attributed to the higher surface area provided by the ultrafine fibrous structure, which facilitated the release of lysozyme into the dissolution medium. Although the overall difference in the release kinetics can be considered marginal, the identification of this occurrence could be achieved successfully.

\section{CONCLUSION}

Fully automated fiber-optic systems provide many advantages for the dissolution testing of electrospun fibers as the high sampling frequency made possible by the in situ fiber optics allows for a deeper insight into release kinetics. Further, the adapter designed in the current work provided the required support for the tested samples and prevented the undesired sample folding. The present study highlights the potential of applying fiber-optic dissolution systems for investigating drug release from electrospun fiber mats, thus providing an advanced alternative to the conventional dissolution experimental setups.

\section{CONFLICT OF INTEREST}

The authors report no conflict of interest.

\section{REFERENCES}

1. Greiner, A.; Wendorff, J. H. Electrospinning: A Fascinating Method for the Preparation of Ultrathin Fibers. Angew. Chem., Int. Ed. Engl. 2007, 46 (30), 5670-5703. DOI: 10.1002/anie.200604646.

2. Sill, T. J.; von Recum, H. A. Electrospinning: applications in drug delivery and tissue engineering. Biomaterials 2008, 29 (13), 1989-2006. DOI: 10.1016/j.biomaterials.2008.01.011.

3. Agarwal, S.; Wendorff, J. H.; Greiner, A. Use of electrospinning technique for biomedical applications. Polymer 2008, 49 (26), 5603-5621. DOI: 10.1016/j.polymer.2008.09.014.

4. Meinel, A. J.; Germershaus, O.; Luhmann, T.; Merkle, H. P.; Meinel, L. Electrospun matrices for localized drug delivery: Current technologies and selected biomedical applications. Eur. J. Pharm. Biopharm. 2012, 81 (1), 1-13. DOI: 10.1016/j.ejpb.2012.01.016.

5. Srikar, R.; Yarin, A. L.; Megaridis, C. M.; Bazilevsky, A. V.; Kelley, E. Desorption-Limited Mechanism of Release from Polymer Nanofibers. Langmuir 2008, 24 (3), 965-974. DOI: 10.1021/la702449k.

6. Martin, C. Evaluating the Utility of Fiber Optic Analysis for Dissolution Testing of Drug Products. Dissolution Technol. 2003, 10 (4), 37-40. DOI: 10.14227/ dt100403p37.

7. Josefson, M.; Johansson, E.; Torstensson, A. Optical Fiber Spectrometry in Turbid Solutions by Multivariate Calibration Applied to Tablet Dissolution Testing. Anal. Chem. 1988, 60 (24), 2666-2671. DOI: 10.1021/ ac00175a004.

8. Bijlani, V.; Yuonayel, D.; Katpally, S.; Chukwumezie, B. N.; Adeyeye, M. C. Monitoring ibuprofen release from multiparticulates: In situ fiber-optic technique versus the HPLC method: A technical note. AAPS PharmSciTech 2007, 8 (3), 9-12. DOI: 10.1208/ pt0803052.

9. Toher, C. J.; Nielsen, P. E.; Foreman, A. S.; Avdeef, A. In Situ Fiber Optic Dissolution Monitoring of Vitamin B12 Solid Dosage Formulation. Dissolution Technol. 2003, 10 (4), 20-25. DOI: 10.14227/dt100403p20.

10. Kenawy, E.; Bowlin, G. L.; Mansfield, K.; Layman, J.; Simpson, D. G.; Sanders, E. H.; Wnek, G. E. Release of tetracycline hydrochloride from electrospun poly(ethylene-co-vinylacetate), poly(lactic acid), and a blend. J. Controlled Release 2002, 81 (1-2), 57-64. DOI: 10.1016/S0168-3659(02)00041-X.

11. 2.9.4. Dissolution Test for Transdermal Patches. In European Pharmacopoeia, 6th ed.; European 
Directorate for the Quality of Medicines \& Healthcare, Council of Europe: Strasbourg, France, 2008.

12. Preis, M.; Woertz, C.; Schneider, K.; Kukawka, J.; Broscheit, J.; Roewer, N.; Breitkreutz J. Design and evaluation of bilayered buccal film preparations for local administration of lidocaine hydrochloride. Eur. J. Pharm. Biopharm. 2014, 86 (3), 552-561. DOI: 10.1016/j.ejpb.2013.12.019.

13. Garsuch, V; Breitkreutz, J. Comparative investigations on different polymers for the preparation of fastdissolving oral films. J. Pharm. Pharmacol. 2010, 62 (4), 539-545. DOI: 10.1211/jpp/62.04.0018.

14. Verreck, G.; Chun, I.; Rosenblatt, J.; Peeters, J.; Van Dijck, A.; Mensch, J.; Noppe, M.; Brewster, M. E. Incorporation of drugs in an amorphous state into electrospun nanofibers composed of a waterinsoluble, nonbiodegradable polymer. J. Controlled Release 2003, 92 (3), 349-360. DOI: 10.1016/S01683659(03)00342-0. 